\title{
640 STEM-LIKE CD4 T CELLS IN CANCER
}

Maria Cardenas*, Nataliya Prokhnevska, Caroline Jansen, Viraj Master, Haydn Kissick. Emory University, Atlanta, GA, United States

Background CD4 $\mathrm{T}$ cells can differentiate into multiple effector subsets that can mediate variable functions. In this work we aim to understand how CD4 $\mathrm{T}$ cells differentiate in response to tumor antigens and their respective function in the anti-tumor response.

Methods Tumor tissue was collected from patients undergoing surgery at Emory University Hospital. Activated PD1+ CD45RA- tumor infiltrating CD4 $\mathrm{T}$ cells were sent for $10 \mathrm{X}$ single cell RNA-seq. Tumor samples were also processed for flow cytometry and ex vivo functional analyses. For in vivo studies, prostate cancer mouse model expressing the LCMV glycoprotein (TRAMPC1-GP) was used, as well as LCMV Armstrong infection.

Results To characterize the heterogeneity of CD4 T cells infiltrating kidney tumors, we performed single cell RNAseq. We found three distinct activated (PD1+ CD45RA-) CD4 T cell populations. Two effector clusters consisting of Th1-like $(\mathrm{EOMES}+)$ and Treg $(\mathrm{FOXP}+)$ cells, and a third cluster expressing TCF1, and genes associated with stemness and survival that did not fit defined CD4 effector lineages. We further confirmed these data by flow cytometry and found the same tumor infiltrating CD4 subsets in 100 kidney cancer patients. When placed in culture under different polarization conditions, tumor TCF1+ CD4 T cells proliferated and differentiated into the Th1-like and Treg effector populations found in the tumor, in addition to other effector lineages (Th1, Tfh) given the appropriate conditions, while the Th1-like and Treg cells underwent no proliferation or phenotype changes. These data suggests that the TCF1+ CD4s act as activated unpolarized precursors to the effector subsets in the tumor. To further test this hypothesis in vivo, we adoptively transferred tumor specific (SMARTA) CD4 T cells into mice followed by TRAMPC1-GP tumor inoculation. Transferred SMARTAs activated and first acquired a TCF1+ phenotype in the TDLN prior to predominantly differentiating into Tregs in the tumor. Given their plasticity in vitro, we asked whether TCF1+ SMARTAs primed in tumors were destined to differentiate into Tregs. To test this, we transferred 4-week activated TCF1 + SMARTAs from TDLNs of TRAMPC1-GP mice into naïve mice that were immediately infected with LCMV Armstrong. We found that the transferred SMARTAs differentiated into Th1 and Tfh cells in response to the virus, similar to the endogenous virus specific CD4 T cells.

Conclusions Overall, this work shows that CD4 T cells remain in an activated phenotype in the tumor with the capacity to differentiate into non-suppressive effector lineages given the appropriate conditions that may benefit the anti-tumor response.

http://dx.doi.org/10.1136/jitc-2021-SITC2021.640 